\title{
An analysis of multifactorial influences on the transcriptional control of ompF and ompC porin expression under nutrient limitation
}

\author{
Xueqiao Liu and Thomas Ferenci
}

Department of

Microbiology G08,

University of Sydney,

Sydney, New South Wales

2006, Australia
Author for correspondence: Thomas Ferenci. Tel: +6129351 4277. Fax: +61293514571.

e-mail: tferenci@mail.usyd.edu.au

Expression of the major outer-membrane porins in Escherichia coli is transcriptionally controlled during nutrient limitation. Expression of ompF was more than 40-fold higher under glucose limitation than under nitrogen (ammonia) limitation in chemostat cultures at the same growth rate. In contrast, ompC expression was higher under $\mathbf{N}$ limitation. The basis of regulation by nutrient limitation was investigated using mutations affecting expression of porin genes. The influence of cyaA, rpoS, ackA and pta, as well as the two-component envZ-ompR system, was studied under glucose and $\mathbf{N}$ limitation in chemostat cultures. A major contributor to low ompF expression under $\mathbf{N}$ limitation was negative control by the RpoS sigma factor. RpoS levels were high under $\mathbf{N}$ limitation and loss of RpoS resulted in a 19-fold increase in ompF transcription, but little change was observed with ompC. Lack of RpoS under glucose limitation had a lesser stimulatory effect on ompF expression. Porin production was minimally dependent on EnvZ under $\mathbf{N}$ limitation due to OmpR phosphorylation by acetyl phosphate. Evidence obtained with pta and ackA mutants suggested that the acetyl phosphate level also regulates porins independently and indirectly via RpoS and other pathways. pta-envZ double mutants had a residual level of porin transcription, implicating alternative means of OmpR phosphorylation under nutrient limitation. Another critical factor in regulation was the level of CAMP, as a cyaA mutant hardly expressed ompF under glucose limitation but boosted ompC. In addition, the role of DNA-binding proteins encoded by hns and himA was tested under glucose limitation: the hns mutation reduced the glucose-limitation peak, but the himA mutation suppressed the hns effect, suggesting a complex web of interrelationships between the DNA-binding proteins. Indeed, multiple inputs and no single regulator were responsible for the high peak of ompF expression under glucose limitation.

Keywords: chemostat culture, Escherichia coli outer-membrane, RpoS, cAMP, acetyl phosphate

\section{INTRODUCTION}

Nutrient availability is a major factor in bacterial survival and proliferation (Nystrom, 1998). Reduced growth rate resulting from nutrient limitation invokes a number of regulated changes in bacterial outer-membrane composition (Lugtenberg et al., 1976; Overbeeke \& Lugtenberg, 1980; Sterkenburg et al., 1984). Porin

Abbreviations: $A C P$, acetyl phosphate; $D$, dilution rate; $\mathrm{H}-\mathrm{NS}$, histone-like DNA-binding protein; IHF, integration host factor. proteins control the permeability of the outer membrane and nutrient limitation strongly and differentially regulates porin expression (Overbeeke \& Lugtenberg, 1980; Liu \& Ferenci, 1998). Aside from nutrient limitation, porin levels are also sensitive to a wide variety of environmental parameters including osmolarity, temperature, $\mathrm{pH}$, growth phase and cell density (Pratt et al., 1996; Buckler et al., 2000). The mechanism of porin regulation is particularly complex and many factors are involved in porin transcriptional and translational control (Pratt et al., 1996). The best-understood input 


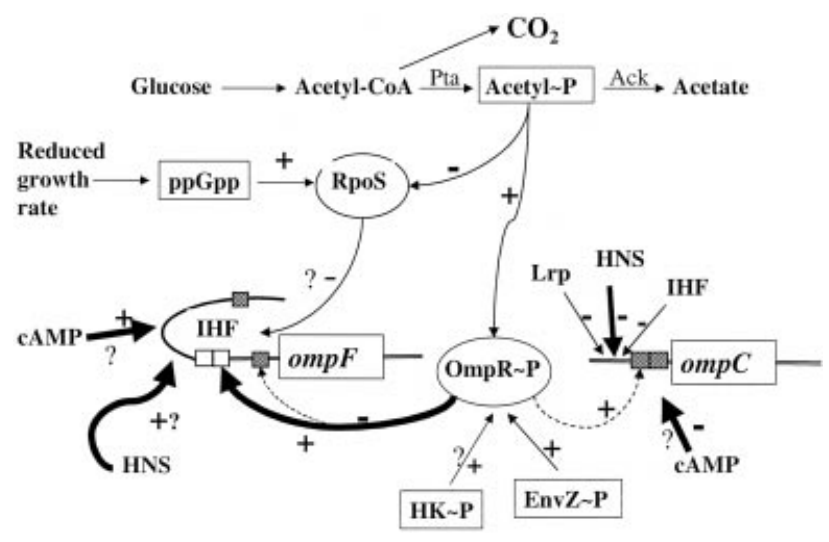

Fig. 1. Transcriptional regulation of porin expression in E. coli. The promoters of the $o m p F$ and $o m p C$ genes include binding sites for OmpR-P with high and low affinity (open and shaded boxes). High ompF expression requires low OmpR-P levels, whereas high ompC expression and repressed ompF requires high OmpR-P (dashed lines; Pratt et al., 1996). Additional regulatory factors can influence porin regulation by affecting the level of OmpR-P; for example, EnvZ, AcP or alternative histidine kinases (HK-P). A second way of affecting regulation is by demonstrated interactions with the respective promoters; for example, IHF at both promoters, H-NS and Lrp at the ompC promoter. A third category of regulators which have no defined molecular mechanism includes RpoS and CAMP (indicated by question marks). Under nutrient limitation with reduced growth rates, ppGpp (Gentry et al., 1993) and the level of AcP influence the level of Rpos (Hengge-Aronis, 2000). Stimulatory and inhibitory effects are designated by $(+)$ and $(-)$, respectively. The major influences operating under glucose limitation are denoted by thick arrows.

into controlling porin transcription involves EnvZ and OmpR (Fig. 1). OmpR phosphorylation is essential for porin transcription: a high level of OmpR-P stimulates $o m p C$ and represses ompF, and a low level of OmpR-P induces ompF transcription (Kenney et al., 1995; Lan \& Igo, 1998; Matsubara \& Mizuno, 1999). EnvZ plays a major role in OmpR phosphorylation and dephosphorylation (Russo \& Silhavy, 1991; Tokishita \& Mizuno, 1994; Kenney, 1997). High osmolarity produces more OmpR-P by increasing EnvZ phosphorylation, while low osmolarity induces the activity of EnvZ phosphatase, resulting in dephosphorylated OmpR-P (Slauch et al., 1988). Porin regulation is, however, not totally dependent on EnvZ (Forst et al., 1988). Several alternative histidine kinase donors (Matsubara \& Mizuno, 1999), and some high-energy molecules including acetyl phosphate $(\mathrm{AcP})$, phosphoramidate and carbamyl phosphate can phosphorylate OmpR (McCleary et al., 1993). Aside from EnvZ and OmpR, many global regulators such as alternative sigma factor $\left(\sigma^{\mathrm{s}}\right.$; Pratt et al., 1996), histone-like DNA-binding protein (H-NS; Atlung \& Ingmer, 1997), integration host factor (IHF; Goosen \& Van de Putte, 1995) and AcP (Heyde et al., 2000) all regulate porin expression at the transcriptional level. Another signal affecting porin regulation by unknown pathways is cAMP ( $\mathrm{S}$ cott \& Harwood, 1980). Additional regulatory circuits affect the control of OmpF translation through MicF (Suzuki et al., 1996), but translational control will not be considered in this study.

Nutrient limitation, resulting in reduced growth rates, launches a wide range of adaptations in both physiological and regulatory circuits. Nutrient limitation enhances nutrient-scavenging ability by improving outer-membrane permeability and inducing high-affinity cytoplasmic-membrane transport systems (Ferenci, 1996, 1999). It also triggers dramatic changes in the intracellular pool of metabolites or the 'metabolome' (Tweeddale et al., 1998). Signalling molecules such as polyphosphate (Kornberg et al., 1999), ppGpp (Gentry et al., 1993), cAMP (Notley-McRobb et al., 1997), AcP (McCleary et al., 1993), anhydroglucitol (Shiga et al., 1999), and endoinducers such as galactose and maltotriose (Death \& Ferenci, 1994; Ferenci, 1996) are all influenced by nutrient limitation. Also, global regulators such as $\sigma^{\mathrm{s}}$ (Notley \& Ferenci, 1996; Teich et al., 1999) are affected by nutrient limitation and in turn affect expression of a large number of genes (Hengge-Aronis, 1999).

We previously reported that porin expression was strongly and differentially regulated by nutrient limitation (Liu \& Ferenci, 1998). Expression of ompF in Escherichia coli was much higher under glucose limitation than under growth limitation by $\mathrm{N}$ source and peaked sharply at glucose concentration below $1 \mu \mathrm{M}$, while ompC expression was higher under $\mathrm{N}$ limitation and repressed by glucose limitation. The expression of both ompF and ompC was shown to be absolutely dependent on OmpR, and loss of ompR abolished ompF and $\operatorname{ompC}$ transcription under both glucose and $\mathrm{N}$ limitation (Liu \& Ferenci, 1998).

A chemostat culture approach was used to study porin regulation in this work because it permits reproducible conditions for nutrient limitation. Briefly, the cultures were grown at a dilution rate of $D=0.3 \mathrm{~h}^{-1}$, corresponding to doubling times of approximately $2.5 \mathrm{~h}$. Cultures growing at $D=0.3 \mathrm{~h}^{-1}$ express a hunger response with a partial elevation in RpoS and associated cellular changes (Ferenci, 1999). This dilution rate was chosen because it is the optimum for $o m p F$ expression in glucose-limited continuous cultures (Liu \& Ferenci, 1998). In N-limited continuous cultures, the low ammonium ion concentration limits growth whereas the glucose concentration remains in the millimolar range. The glucose-excess conditions of N-limited cultures have different metabolic and regulatory consequences; for example, acetate production is high in $\mathrm{N}$ limited, but low in glucose-limited chemostats (el-Mansi \& Holms, 1989). In contrast, cAMP levels are low in Nlimited but high in glucose-limited cultures (NotleyMcRobb et al., 1997). Also, as shown in this study, RpoS levels are higher under $\mathrm{N}$ limitation than under glucose limitation. The influence of these and other factors on porin regulation is considered below.

Porin regulation under nutrient limitation is complex and poorly understood. The simplest working hypoth- 
Table 1. Bacterial strains used in this study

\begin{tabular}{|c|c|c|}
\hline Strain & Relevant genotype & Source/reference \\
\hline BW2800 & $\operatorname{MC} 4100 / \mathrm{F}^{\prime}\left(\operatorname{lac} I^{\mathrm{q} 1}, \operatorname{lac} Z:: \operatorname{Tn} 5, \operatorname{lac} \mathrm{Y}^{+}, \operatorname{lac}^{+}\right)$ & Ferenci \& Stretton (1989) \\
\hline BW3301 & MH513 rpoS:: Tn10 & This study \\
\hline BW3302 & MH225 rpoS:: Tn10 & This study \\
\hline BW3303 & MH513 ompR:: Tn10 & This study \\
\hline BW3304 & MH225 ompR:: Tn10 & This study \\
\hline BW3305 & MH513 hns::neo & This study \\
\hline BW3306 & MH225 hns::neo & This study \\
\hline BW3308 & MH513 himA $\Delta 82$ tet & This study \\
\hline BW3309 & MH225 himAs82tet & This study \\
\hline BW3319 & BW3305 rpos: : Tn10 & This study \\
\hline BW3320 & BW3306 rpoS:: Tn10 & This study \\
\hline BW3321 & BW3305 himA $\Delta 82:$ :tet & This study \\
\hline BW3322 & BW3306 himA $22:$ :tet & This study \\
\hline BW3323 & MC4100 rpoS:: Tn10 & This study \\
\hline BW3329 & BW3301 hns::neo & This study \\
\hline BW3343 & MH513 envZ60:: $\operatorname{Tn} 10$ & This study \\
\hline BW3345 & MH225 envZ60:: Tn10 & This study \\
\hline BW3346 & BW3343 ackA:: $\operatorname{Tn} p h o A^{\prime}-9$ & This study \\
\hline BW3357 & MH513 ackA:: TnphoA'-9 & This study \\
\hline BW3358 & MH225 ackA:: TnphoA'-9 & This study \\
\hline BW3366 & BW3358 envZ60:: Tn10 & This study \\
\hline BW3367 & BW3358 rpoS: : Tn10 & This study \\
\hline BW3368 & BW3357 rpos:: $\operatorname{Tn} 10$ & This study \\
\hline BW3381 & DY330 cyaA:: tet & This study \\
\hline BW3382 & DY330 pta::kan & This study \\
\hline BW3383 & MH513 cyaA:: tet & This study \\
\hline BW3601 & MH513 pta::kan & This study \\
\hline BW3602 & MH225 pta::kan & This study \\
\hline BW3603 & BW3345 pta: :kan & This study \\
\hline BW3605 & MH225 cyaA:: tet & This study \\
\hline BW3607 & BW3343 pta: :kan & This study \\
\hline BW3608 & BW3601 rpoS: : Tn10 & This study \\
\hline BW3609 & BW3602 rpos:: $\operatorname{Tn} 10$ & This study \\
\hline СР724 & ack $A:: \operatorname{Tn} p h o A^{\prime}-9$ & Shin \& Park (1995) \\
\hline CU221 & $\mathrm{CSH} 26 \Delta($ pro-lac $)$ ara thi hns::neo & Yamada et al. (1991) \\
\hline CV1008 & $\mathrm{F}^{-}$ara thi $\Delta l a c$ pro ilvIH: : Mudl1734 lrp:: Tn10 & M. Freundlich* \\
\hline DY330 & W3110 $\Delta$ lacU169 gal490 $\lambda$ cl857 $\Delta($ cro-bioA $)$ & Yu et al. (2000) \\
\hline HN1641 & W3102 (galK2) supO strR himA & H. Nash* \\
\hline JB100 & HfrG6 malT ${ }^{\mathrm{C}}-1$ ompR-Tn10 & Brass et al. (1984) \\
\hline JMS6210 & envZ60:: Tn10 recA111::Kan & Slauch et al. (1988) \\
\hline MC4100 & $\begin{array}{l}\mathrm{F}^{-} \text {araD139 } \Delta(\operatorname{argF}-\text { lac }) \text { U169 rpsL150 deoC1 relA1 } \\
\text { thiA ptsF25 flbB530 rbsR }\end{array}$ & Casabadan (1976) \\
\hline $\begin{array}{l}\text { MC4100 } \\
\Delta \text { fis }\end{array}$ & MC4100 $\Delta$ fis: : cat & H. Nash \\
\hline MH225 & MC4100 $\Phi\left(\right.$ ompC-lac $\left.Z^{+}\right) 10-25$ & Hall \& Silhavy (1981) \\
\hline MH513 & MC4100 araD ${ }^{+} \Phi\left(o m p \mathrm{~F}^{\prime}-l a c Z^{+}\right) 16-13$ & Hall \& Silhavy (1981) \\
\hline ZK1171 & W3110 slacU169 tna-2 rpoS: :Tn10 & Huisman \& Kolter (1994) \\
\hline
\end{tabular}

*M. Freundlich, Dept. of Biochemistry and Cell Biology, State University of New York, Stony Brook, NY 11794, US; H. Nash, NIMH-LMB, Bethseda, MD 20892, US.

esis to explain the nutrient-limitation data was that the induction of $o m p F$ under glucose limitation was due to the low level of OmpR-P (optimal for ompF, and not ideal for ompC; Lan \& Igo, 1998). The repression of
ompF and induction of $o m p C$ by $\mathrm{N}$ limitation is then explicable by the high level of OmpR-P. To test this simple hypothesis, the studies described below explored regulation under nutrient limitation by detailed analysis 
of the influence of the two-component envZ-ompR system. As shown in Fig. 1, other factors such as AcP, rpoS, cyaA (via cAMP) and DNA-binding proteins such as H-NS and IHF influence porin expression, so these inputs were also investigated. The hypothesis that OmpR phosphorylation was the central factor in porin regulation proved to be only partly substantiated, and we provide evidence of the importance of AcP, cAMP and $\mathrm{RpoS}$ in porin gene expression under nutrient limitation.

\section{METHODS}

Bacterial strains and culture conditions. Bacterial strains used in this study are listed in Table 1. P1 transduction was used to construct mutants (Miller, 1972). The media and growth conditions for glucose- and ammonia-limited chemostats and batch culture were as previously described (Death et al., 1993; Liu \& Ferenci, 1998). The total salt concentration was approximately $100 \mathrm{mM}$, so provided a high-osmolarity environment.

Construction of cyaA::tet and pta::kan insertions. The insertion of antibiotic-resistance cassettes in the cyaA and pta genes was accomplished using the method of Yu et al. (2000). Briefly, linear tet and kan cassettes were amplified by PCR from strains BW3303 and BW2800 respectively, and recombined into the cyaA and pta genes in DY330 (Yu et al., 2000) after electroporation. The cassettes in the resulting drugresistant strains BW3381 and BW3382 were checked by PCR using primers flanking the target gene. The size of the gene with insertions was compared with the size of the original gene on a $1 \%$ agarose gel. After confirmation of the insertion, the mutated gene was picked up by $\mathrm{P} 1$ phage and transferred to the recipients (shown in Table 1).

For construction of the $c y a A:$ : tet mutation, the primers used to amplify tet were $5^{\prime}$-CCTATTCCTGGGAATACCCGAACCCACGTCCAAGAGGGTCATTATATTTCG-3' and ${ }^{\prime}{ }^{\prime}-$ GACGTGATTGGTTTCAACCTGCACTGACAGGACTCGACATCTTGGTTACCG-3'. For construction of the pta::kan mutant, the primers used to amplify kan were $5^{\prime}$ CGACTATCGTGCGTGCGAACTCTTCCACCACTATGGACAGCAAGC GAACCG-3' and 5'-GCACAGATAGCGGCTGCTTTAACGGTACGCGTCAGAAGAACTCGTCAAGAAG-3'.

$\boldsymbol{\beta}$-Galactosidase and RpoS protein assays. The $\beta$-galactosidase activity of lac $Z$ fusions of bacteria grown in the chemostat for $3 \mathrm{~d}$ was assayed, as described by Miller (1972), with results expressed in Miller units. The $\beta$-galactosidase activities presented in Table 2 and Table 3 were the mean of three to five separate chemostat culture results. The amount of RpoS was analysed by Western blotting. The procedures for protein extraction, SDS-PAGE separation, electrophoretic transfer and immunodetection of RpoS were as previously described (Liu et al., 2000), and as specified in the legends.

\section{RESULTS AND DISCUSSION}

\section{OmpR and EnvZ influence on porin expression under nutrient limitation}

As previously reported (Liu \& Ferenci, 1998), the transcriptional activator $\mathrm{OmpR}$ was needed for porin expression under nutrient limitation. OmpR control of porin gene transcription by osmolarity involves phosphorylation and dephosphorylation by EnvZ (Pratt et al., 1996). The contribution of EnvZ to expression under nutrient limitation, but at constant osmolarity, was therefore analysed in the presence of an envZ60::Tn10 mutation under both glucose and $\mathrm{N}$ limitation (Table 2). Under glucose limitation, disruption of envZ partially decreased expression of both $o m p F-l a c Z$ and ompC-lacZ transcriptional fusions. Nevertheless, there was still significant EnvZ-independent transcription of $o m p F$ and $o m p C$ under glucose limitation, above that seen with the very low level in the ompR:: Tn10 mutant in glucose- and N-limited chemostats (Table 2).

Even more interestingly, the expression of $o m p F$ and ompC in envZ mutants was as high, or higher, under $\mathrm{N}$ limitation than found with wild-type envZ. The high residual ompC expression in envZ mutants suggested there was a considerable level of OmpR phosphorylation under glucose-rich conditions in the absence of EnvZ. The absence of EnvZ-dependent dephosphorylation may also have contributed to elevated OmpR-P levels. These results confirm that $\mathrm{OmpR}$ is a central regulator (as shown in Fig. 1) under both glucose and $\mathrm{N}$ limitation, but the role of EnvZ may not be essential under glucoserich conditions.

\section{RpoS influence on porin expression}

The sigma factor RpoS has a negative effect on ompF expression in stationary phase (Pratt et al., 1996), so its role under steady-state nutrient limitation was tested. Table 2 shows the influence of an $r p o S:: \operatorname{Tn} 10$ mutation on the transcription of $o m p F$ and $o m p C$ under glucose and $\mathrm{N}$ limitation. Disruption of the rpoS gene caused enhanced expression of ompF transcription under both glucose and $\mathrm{N}$ limitation, in line with negative control by RpoS (Fig. 1). The effect was particularly marked under $\mathrm{N}$ limitation, with a remarkable 19 -fold increase at $D=0 \cdot 3 \mathrm{~h}^{-1}$. This result suggested that RpoS levels must be particularly high under $\mathrm{N}$ limitation to repress $o m p F$ expression to such a large extent in the wild-type. The quantity of RpoS under $\mathrm{N}$ limitation was not previously assayed but is shown in the Western blot in Fig. 2. The level of RpoS protein under glucose and $\mathrm{N}$ limitation shows that growth rate as well as nutrient availability affect the concentration of this protein. Data obtained from densitometer scans showed that $\mathrm{N}$ limitation produced more RpoS protein than glucose limitation, with fourfold more RpoS protein detected at $D=0.3 \mathrm{~h}^{-1}$ than under glucose limitation. Evidence from mutants and the protein assays together suggest that the level of RpoS had a significant repressive effect on ompF transcription in $\mathrm{N}$-limited environments.

A mutation in rpoS did not influence ompC expression nearly as much as that of $o m p F$ under either glucose or $\mathrm{N}$ limitation. Glucose limitation gave little difference in ompC expression at $D=0.3 \mathrm{~h}^{-1}$. Under $\mathrm{N}$ limitation, there was a small increase in the rpoS mutant, possibly due to competition between sigma factors when RpoS is high in the wild-type (Farewell et al., 1998). The ompC 
Table 2. Transcriptional regulation of ompF and ompC under glucose and nitrogen limitation growing at $0.3 \mathrm{~h}^{-1}$ dilution rate

\begin{tabular}{|llcc|}
\hline Strain & Relevant genotype & \multicolumn{2}{c|}{$\boldsymbol{\beta}$-Galactosidase activity \pm SD } \\
& & Glucose limitation & Nitrogen limitation \\
& & & \\
\hline MH513 & ompF-lacZ & $2294 \pm 195$ & $51 \pm 3$ \\
BW3303 & MH513 ompR & $6 \cdot 8 \pm 1 \cdot 1$ & $6 \pm 1 \cdot 8$ \\
BW3343 & MH513 envZ60 & $592 \pm 16$ & $60 \pm 2$ \\
BW3357 & MH513 ackA & $2636 \pm 181$ & $1378 \pm 42$ \\
BW3346 & MH513 ackA envZ60 & $2125 \pm 27$ & $1297 \pm 56$ \\
BW3301 & MH513 rpoS & $2559 \pm 186$ & $964 \pm 83$ \\
BW3368 & MH513 ackA rpoS & $2244 \pm 42$ & $1268 \pm 56$ \\
BW3601 & MH513 pta & $973 \pm 14$ & $178 \pm 8$ \\
BW3607 & MH513 envZ60 pta & $280 \pm 72$ & $73 \pm 6$ \\
BW3608 & MH513 pta rpoS & $2426 \pm 24$ & $745 \pm 19$ \\
BW3383 & MH513 cyaA & $65 \pm 5$ & $60 \pm 4$ \\
BW3305 & MH513 hns & $390 \pm 83$ & $118 \pm 27$ \\
MH225 & ompC-lacZ & $116 \pm 33$ & $170 \pm 41$ \\
BW3304 & MH225 ompR & $7 \pm \pm 2$ & $6 \cdot 6 \pm 1 \cdot 7$ \\
BW3345 & MH225 envZ60 & $31 \pm 10$ & $241 \pm 5$ \\
BW3358 & MH225 ackA & $546 \pm 11$ & $603 \pm 58$ \\
BW3366 & MH225 ackA envZ60 & $428 \pm 44$ & $686 \pm 60$ \\
BW3302 & MH225 rpoS & $130 \pm 23$ & $366 \pm 40$ \\
BW3367 & MH225 ackA rpoS & $462 \pm 14$ & $898 \pm 13$ \\
BW3602 & MH225 pta & $515 \pm 85$ & $422 \pm 41$ \\
BW3609 & MH225 pta rpoS & $598 \pm 64$ & $686 \pm 54$ \\
BW3603 & MH225 envZ60 pta & $58 \pm 20$ & $877 \pm 24$ \\
BW3605 & MH225 cyaA & $638 \pm 18$ & \\
BW3306 & MH225 hns & $2100 \pm 80$ & \\
\hline
\end{tabular}

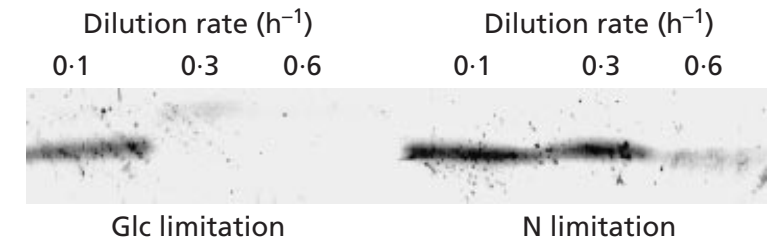

Fig. 2. Levels of RpoS under nutrient limitation. Immunoblotting using anti-RpoS antibodies was performed as described by Liu et al. (2000). Equivalent numbers of bacteria from glucose- and N-limited chemostats were extracted by the SDSboiling method, from chemostats run at the dilution rates shown. Consistent with earlier studies (Notley \& Ferenci, 1996; Teich et al., 1999), the RpoS in glucose-limited chemostats was elevated significantly at the slowest growth rate. The $\mathrm{N}$-limited cultures had 4-fold and 3.6-fold higher levels, as determined by densitometry (Liu et al., 2000), than glucose-limited chemostats at $D=0.3$ and $0.6 \mathrm{~h}^{-1}$, respectively. Protein levels were quantitated from densitometer scans using ImageQuant software in three separate blots, each containing samples from separate chemostat cultures of MC4100 growing at dilution rates of $0 \cdot 1,0.3$ and $0.6 \mathrm{~h}^{-1}$.

results are consistent with the view that $r p o S$ has a much stronger role in $o m p F$ than in $o m p C$ regulation. The mechanism by which RpoS affects transcription from the $o m p F$ promoter more than from ompC is not known.

\section{The dual importance of ACP in porin expression}

Given the high level of acetate metabolism in N-limited cultures (el-Mansi \& Holms, 1989), and the possibility of AcP-dependent OmpR phosphorylation (Kenney et al., 1995), the role of acetate metabolism in porin regulation was investigated. The production of AcP from glucose is controlled by two enzymes: phosphotransacetylase (product of gene $p t a$ ) and acetate kinase (product of ackA) (Fig. 1). Mutation of $p t a$ reduces AcP production from glucose and mutation of ack $A$ prevents AcP breakdown to acetate. AcP was shown to influence the control of porin expression under low-pH conditions (Heyde et al., 2000). To clarify the importance of AcP in porin regulation, the influence of ackA and $p t a$ mutations on ompF and ompC expression was assayed under glucose and $\mathrm{N}$ limitation.

The ackA mutation blocking AcP breakdown strongly influenced porin expression under both glucose and $\mathrm{N}$ limitation. The expression of ompC (dependent on high OmpR-P levels) was increased about four- to fivefold under both glucose and $\mathrm{N}$ limitation (Table 2). Under $\mathrm{N}$ limitation, the increase was in addition to the rise with $r p o S$, as shown with the ackA rpoS double mutant. This was not surprising, since RpoS does not influence $o m p C$ directly. Less predictably, the ackA mutation also enhanced the expression of $o m p F$ under both glucose 
and $\mathrm{N}$ limitation. The effect on $o m p F$ expression under glucose limitation was relatively small, but the mutation of ack $A$ increased ompF expression under $\mathrm{N}$ limitation 27-fold.

Given that high OmpR-P levels (derived from extra $\mathrm{AcP}$ ) should result in high ompC but low ompF expression, the increased $o m p F$ expression under $\mathrm{N}$ limitation due to the ackA mutation cannot be explained purely through OmpR-P levels. The ackA results indicated that the accumulation of AcP must have altered control of other factors such as RpoS (which is involved in control of porin regulation; Table 2); AcP is already known to negatively affect RpoS levels (Bouche et al., 1998). A comparison of the results in Table 2 between $r p o S$ and ackA mutants shows that the pattern of expression of $o m p F$ in these mutants is similar under both glucose and $\mathrm{N}$ limitation. This resemblance suggests that high ompF expression with high $\mathrm{AcP}$ is an indirect effect of reduction of RpoS levels. To see if the two mutations were epistatic, the influence of further introducing the rpoS mutation into the ackA strains was examined (Table 2). The double mutation of ackA and rpoS resulted in almost the same expression of $o m p F$ as that of the $r p o S$ single mutation under glucose limitation. A complication is that the ackA mutation had a greater effect on ompF expression under $\mathrm{N}$ limitation than the rpoS mutation; this leaves open the possibility that AcP levels in porin regulation influence another factor besides RpoS.

To clarify the contribution of EnvZ and AcP to OmpR$\mathrm{P}$ formation, an envZ ackA double mutant was constructed and ompF/ompC expression retested. The additional mutation in env $Z$ had little influence on $o m p F$ and $\operatorname{ompC}$ expression in either glucose- or Nlimited cultures. These results suggest EnvZ may be not an essential contributor to OmpR phosphorylation, particularly under $\mathrm{N}$ limitation, and $\mathrm{AcP}$ has a significant role in the phosphorylation of OmpR leading to high ompC expression. Nevertheless, the loss of OmpR$\mathrm{P}$ phosphatase in an env $\mathrm{Z}$ mutant could also contribute to high OmpR-P levels in the double mutant.

\section{Influence of a pta mutation on porin expression}

In contrast to the ack $A$ mutation, the pta mutation blocks AcP synthesis from glucose. The influence of decreased AcP on porin expression was checked with the pta mutants shown in Table 2.

The pta mutation resulted in a reduction of ompF expression under glucose limitation. Two explanations for this decrease were possible. The first possibility was that OmpR-P is lowered due to lack of AcP so there is insufficient OmpR-P for ompF expression. This was unlikely because ompC expression, requiring higher OmpR-P, was actually higher in the pta mutant. The second possibility was that lack of AcP in the pta mutant led to higher levels of RpoS and hence repression of $o m p F$ (Fig. 1). This is more likely, given that an rpoS mutation restored ompF expression in the $p t a$ rpoS double mutant. The properties of the pta mutants suggest $\mathrm{AcP}$ has a dual regulatory role under nutrient limitation, via both OmpR and RpoS.

The higher ompC expression in the pta mutant was unexpected and not explicable solely in terms of AcP acting as a phosphoryl donor to supply OmpR-P. The increase was entirely dependent on envZ-mediated OmpR phosphorylation, as the pta env $Z$ double mutant had low ompC expression (Table 2). The pta mutation by itself must somehow indirectly change porin regulation through unknown mechanisms. We speculate that a lack of AcP may increase EnvZ-kinase activity to explain our results.

Under $\mathrm{N}$ limitation, expression of $o m p F$ was increased by the pta mutation. This was possibly due to the reduced availability of AcP lowering the OmpR-P level closer to that optimal for $o m p F$ expression; however, this is not the case because, surprisingly, the pta::kan mutation significantly increased ompC expression in both glucose- and N-limited cultures. Hence, a level of OmpR-P stimulating ompC expression should be too high for elevated ompF expression. The lack of AcP should also lead to higher RpoS levels, leading to ompF repression. More likely, the lack of AcP has more complex indirect effects than the ones considered so far. For example, indirect (and as yet unexplored) effects on other components in Fig. 1 or on DNA supercoiling (Graeme-Cook et al., 1989) could explain complex patterns of $o m p F$ and $o m p C$ expression changes.

The expression of ompF was also checked with pta envZ double mutants, in which the absence of OmpR phosphorylation by AcP and EnvZ should lead to low porin expression. Compared to a pta single mutation, the pta envZ double mutant reduced ompF expression under both glucose and $\mathrm{N}$ limitation but there was still at least 10-fold higher expression than seen with the ompR mutant. In contrast, the expression of ompC showed a greater decrease under both glucose and $\mathrm{N}$ limitation, as would be expected from a greater need for high OmpR-P levels than for ompF.

\section{Influence of adenylate cyclase on porin expression}

An early study implicated cAMP in porin regulation (Scott \& Harwood, 1980). Given that cAMP levels are much higher under glucose than $\mathrm{N}$ limitation (Death \& Ferenci, 1994), the role of $c y a A$ under nutrient limitation was investigated. Unfortunately, crp mutants could not be studied here as they did not grow well under glucose limitation except at slow dilution rates (results not shown).

The lack of cAMP in the cyaA mutant led to a drastic drop of ompF expression under glucose limitation, but not $\mathrm{N}$ limitation. This result was in line with the relative amounts of cAMP under the two forms of limitation. It also underlined the significance of cAMP to the large peak of ompF expression under glucose limitation.

Expression of $o m p C$ was increased significantly in the cyaA mutant under both glucose and $\mathrm{N}$ limitation. This was consistent with earlier findings in batch culture, 
Table 3. Contribution of DNA-binding proteins to porin transcription under glucose limitation at $0.3 \mathrm{~h}^{-1}$ dilution rate

\begin{tabular}{|llc|}
\hline Strain & Relevant genotype & $\begin{array}{c}\boldsymbol{\beta} \text {-Galactosidase } \\
\text { activity } \pm \text { SD }\end{array}$ \\
\hline MH513 & ompF-lacZ & $2294 \pm 195$ \\
BW3308 & MH513 himA & $2136 \pm 27$ \\
BW3305 & MH513 hns & $390 \pm 83$ \\
BW3319 & MH513 hns rpoS & $445 \pm 31$ \\
BW3321 & MH513 himA hns & $1609 \pm 47$ \\
MH225 & ompC-lacZ & $116 \pm 33$ \\
BW3309 & MH225 himA & $476 \pm 108$ \\
BW3306 & MH225 hns & $2100 \pm 80$ \\
BW3320 & MH225 hns rpoS & $1700 \pm 62$ \\
BW3322 & MH225 himA hns & $2050 \pm 123$ \\
\hline
\end{tabular}

showing that the addition of cAMP repressed ompC expression (Scott \& Harwood, 1980; Thomas \& Booth, 1992). The mechanistic connection between cAMP and porin expression is still poorly understood, as there is no obvious role of cAMP/Crp in porin transcription directly (Pratt et al., 1996). The influence of cAMP on porin expression may be indirect and through other factors such as OmpR or RpoS. cAMP/Crp can bind to the promoter region of the ompB operon and can both positively and negatively affect expression from the ompR/envZ promoter (Huang et al., 1992). It is unlikely that mutation of $c y a A$ decreases OmpR (and therefore decreases OmpR-P) because expression of ompC increased under both conditions, and the increased ompC requires high levels of OmpR-P (Slauch \& Silhavy, 1989). The simplest explanation for the decrease in $o m p F$ and increase in $o m p C$ expression in the absence of cAMP is that a lack of cAMP induces OmpR and results in more OmpR-P, and the high level of OmpR-P represses ompF and induces ompC. Another possible contributor was that decreased cAMP increases RpoS levels (cAMP has been shown to negatively regulate rpoS transcription; Lange \& Hengge-Aronis, 1994). The cyaA mutation could affect ompF expression via elevated levels of RpoS.

\section{DNA-binding proteins and their influence on porin expression under glucose limitation}

The DNA-binding protein H-NS and several other nucleoid-associated proteins, such as HU, IHF, Fis and Lrp act as global regulators in the control of porin gene expression (Tsui et al., 1988; Ferrario et al., 1995; Suzuki et al., 1996; Painbeni et al., 1997). Also, H-NS has a strong influence on RpoS levels (Hengge-Aronis, 1999), which could in turn influence porin expression as indicated above. Therefore, mutants with insertions in these genes were checked for porin gene transcription to test whether they influenced the very high level of ompF expression under glucose limitation (Table 3). The Lrp and Fis inactivation mutants had little effect on regu- lation by nutrient limitation, so are not considered here (results not shown).

The DNA-binding protein IHF is implicated in porin control because it binds upstream of the $\operatorname{ompB}$, ompF and $o m p C$ promoters, resulting in decreased transcription from each of these genes (Ramani et al., 1992). The influence of $\operatorname{bim} A$ on porin transcription was studied and the result is shown in Table 3 . The mutation of himA had surprisingly little effect on ompF, but resulted in a slight increase of ompC expression. This suggested that IHF repression of $o m p F$ was essentially absent under glucose limitation whereas it was still influencing the ompC promoter.

\section{Role of H-NS in porin regulation}

An hns mutation had a huge positive effect on ompC expression, as was expected from the known influence of H-NS on $o m p C$ transcription, i.e. by binding to the intergenic region between $o m p C$ and $m i c F$, resulting in repression of both genes (Suzuki et al., 1996). More surprisingly, there was a marked reduction in ompF expression with the hns mutant. Unlike the situation with ompC, no in vitro evidence exists to indicate that $\mathrm{H}-\mathrm{NS}$ has a binding site in the ompF promoter region and positively regulates $o m p F$ transcription. A possible explanation for this reduction could have been increased RpoS repression with an indirect influence of hns on ompF transcription. H-NS inhibits rpoS expression at both translational and RpoS stability levels (Lange \& Hengge-Aronis, 1994). Therefore, an rpoS mutation was introduced into the hns mutant. The double mutant did not have restored high levels of $o m p F$ expression, ruling out the involvement of RpoS. The complex nature of the hns effect on ompF was suggested by the observation that the double mutation of $\operatorname{him} A$ and hns restored ompF expression close to the level found in the $\operatorname{him} A$ single mutation. These results indicate that the bns loss did not cause reduced ompF expression directly; more likely, HNS may result in elevated IHF or reduce ompF expression in some unclear way.

\section{Conclusions}

The complex web of interactions affecting porin regulation under nutrient limitation is outlined in Fig. 1. RpoS, AcP and cAMP were all significant inputs in differentiating glucose- and N-limited cultures. RpoS was a significant factor in regulation, particularly under $\mathrm{N}$ limitation, where its level was especially high. AcP contributed to regulation as a phosphoryl donor to OmpR, bypassing the EnvZ requirement under $\mathrm{N}$ limitation. A secondary role of AcP was in controlling RpoS levels, indirectly influencing ompF expression. The $c y a \mathrm{~A}$ product was essential for ompF expression under glucose limitation and was confirmed as playing a significant role in the repression of ompC. Lastly, the his and $\operatorname{him} A$ mutations implicated the significance of histone-like proteins in maintaining the wild-type levels of porin production under nutrient limitation. Each of the above involvements leads to unanswered questions 
as to the molecular mechanisms of these effects. Indeed, the means whereby RpoS, cAMP and H-NS influence ompF expression without an obvious interaction with the promoter remains a puzzle and a target of future research. In addition, other factors not considered here, such as DNA supercoiling, could affect transcription (Graeme-Cook et al., 1989). There are other unresolved puzzles as well; for example, the elevated expression of both $o m p F$ and $o m p C$ in the ackA mutant is not easily explicable via the model in Fig. 1.

\section{ACKNOWLEDGEMENTS}

We thank Dr C. Park, Dr C. Ueguchi, Dr M. Freundlich, Dr Daiguan Yu, Dr J. Brass, Dr H. Nash, Dr T. Silhavy, Dr L. Kenney and Dr R. Kolter for their kind provision of strains. We also thank Dr R. Hengge-Aronis for $\sigma^{\mathrm{s}}$ antibody, and Lucinda Notley-McRobb for her consistent help during this research. This research was supported by the Australian Research Council.

\section{REFERENCES}

Atlung, T. \& Ingmer, H. (1997). H-NS - a modulator of environmentally regulated gene expression. Mol Microbiol 24, 7-17.

Bouche, S., Klauck, E., Fischer, D., Lucassen, M., Jung, K. \& Hengge-Aronis, R. (1998). Regulation of RssB-dependent proteolysis in Escherichia coli: a role for acetyl phosphate in a response regulator-controlled process. Mol Microbiol 27, 787-795.

Brass, J. M., Manson, M. D. \& Larson, T. J. (1984). Transposon Tn10-dependent expression of the lamB gene in Escherichia coli. J Bacteriol 159, 93-99.

Buckler, D. R., Anand, G. S. \& Stock, A. M. (2000). Responseregulator phosphorylation and activation: a two-way street? Trends Microbiol 8, 153-156.

Casabadan, M. J. (1976). Transposition and fusion of the lac operon to selected promoters in E. coli using bacteriophage Lambda and Mu. J Mol Biol 104, 541-555.

Death, A. \& Ferenci, T. (1994). Between feast and famine: endogenous inducer synthesis in the adaptation of Escherichia coli to growth with limiting carbohydrates. J Bacteriol 176, 5101-5107.

Death, A., Notley, L. \& Ferenci, T. (1993). Derepression of LamB protein facilitates outer membrane permeation of carbohydrates into Escherichia coli under conditions of nutrient stress. J Bacteriol 175, 1475-1483.

Farewell, A., Kvint, K. \& Nystrom, T. (1998). Negative regulation by RpoS: a case of sigma factor competition. Mol Microbiol 29, 1039-1051.

Ferenci, T. (1996). Adaptation to life at micromolar nutrient levels: the regulation of Escherichia coli glucose transport by endoinduction and cAMP. FEMS Microbiol Rev 18, 301-317.

Ferenci, T. (1999). Regulation by nutrient limitation. Curr Opin Microbiol 2, 208-213.

Ferenci, T. \& Stretton, S. (1989). Cysteine-22 and cysteine-38 are not essential for the functions of maltoporin (LamB protein). FEMS Microbiol Lett 52, 335-339.

Ferrario, M., Ernsting, B. R., Borst, D. W., Wiese, D. E., II, Blumenthal, R. M. \& Matthews, R. G. (1995). The leucineresponsive regulatory protein of Escherichia coli negatively regulates transcription of $o m p C$ and $m i c F$ and positively regulates translation of ompF. J Bacteriol 177, 103-113.
Forst, S., Delgado, J., Ramakrishnan, G. \& Inouye, M. (1988). Regulation of ompC and ompF expression in Escherichia coli in the absence of envZ. J Bacteriol 170, 5080-5085.

Gentry, D. R., Hernadez, V. J., Nguyen, L. H., Jensen, D. B. \& Cashel, M. (1993). Synthesis of stationary-phase sigma factor Sigma-S is positively regulated by ppGpp. J Bacteriol 175, 7982-7989.

Goosen, N. \& Van de Putte, P. (1995). The regulation of transcription initiation by integration host factor. Mol Microbiol $16,1-7$.

Graeme-Cook, K. A., May, G., Bremer, E. \& Higgins, C. F. (1989). Osmotic regulation of porin expression: a role for DNA supercoiling. Mol Microbiol 3, 1287-1294.

Hall, M. N. \& Silhavy, T. J. (1981). The ompB locus and the regulation of the major outer membrane porin proteins of Escherichia coli K12. J Mol Biol 146, 23-43.

Hengge-Aronis, R. (1999). Interplay of global regulators and cell physiology in the general stress response of Escherichia coli. Curr Opin Microbiol 2, 148-152.

Hengge-Aronis, R. (2000). The general stress response in Escherichia coli. In Bacterial Stress Responses, pp. 161-178. Edited by R. Hengge-Aronis \& K. Storz. Washington DC: American Society for Microbiology.

Heyde, M., Laloi, P. \& Portalier, R. (2000). Involvement of carbon source and acetyl phosphate in the external-pH-dependent expression of porin genes in Escherichia coli. J Bacteriol 182, 198-202.

Huang, L., Tsui, P. \& Freundlich, M. (1992). Positive and negative control of $o m p B$ transcription in Escherichia coli by cyclic AMP and the cyclic AMP receptor protein. J Bacteriol 174, 664-670.

Huisman, G. W. \& Kolter, R. (1994). Sensing starvation: a homoserine lactone-dependent signaling pathway in Escherichia coli. Science 265, 537-539.

Kenney, L. J. (1997). Kinase activity of EnvZ, an osmoregulatory signal transducing protein of Escherichia coli. Arch Biochem Biophys 346, 303-311.

Kenney, L. J., Bauer, M. D. \& Silhavy, T. J. (1995). Phosphorylation-dependent conformational changes in OmpR, an osmoregulatory DNA-binding protein of Escherichia coli. Proc Natl Acad Sci U S A 92, 8866-8870.

Kornberg, A., Rao, N. N. \& Ault-Riche, D. (1999). Inorganic polyphosphate: a molecule of many functions. Annu Rev Biochem 68, 89-125.

Lan, C. Y. \& Igo, M. M. (1998). Differential expression of the $\mathrm{OmpF}$ and $\mathrm{OmpC}$ porin proteins in Escherichia coli $\mathrm{K}-12$ depends upon the level of active OmpR. J Bacteriol 180, 171-174.

Lange, R. \& Hengge-Aronis, R. (1994). The cellular concentration of the sigma-S subunit of RNA polymerase in Escherichia coli is controlled at the levels of transcription, translation and protein stability. Genes Dev 8, 1600-1612.

Liu, X. \& Ferenci, T. (1998). Regulation of porin-mediated outer membrane permeability by nutrient limitation in Escherichia coli. J Bacteriol 180, 3917-3922.

Liu, X., Ng, C. \& Ferenci, T. (2000). Global adaptations resulting from high population densities in Escherichia coli cultures. J Bacteriol 182, 4158-4164.

Lugtenberg, B., Peters, R., Bernheimer, H. \& Berendsen, W. (1976). Influence of cultural conditions and mutations on the composition of the outer membrane proteins of Escherichia coli. Mol Gen Genet 147, 251-262.

McCleary, W. R., Stock, J. B. \& Ninfa, A. J. (1993). Is acetyl 
phosphate a global signal in Escherichia coli? J Bacteriol 175, 2793-2798.

el-Mansi, E. M. \& Holms, W. H. (1989). Control of carbon flux to acetate excretion during growth of Escherichia coli in batch and continuous cultures. J Gen Microbiol 135, 2875-2883.

Matsubara, M. \& Mizuno, T. (1999). EnvZ-independent phosphotransfer signaling pathway of the OmpR-mediated osmoregulatory expression of $\mathrm{OmpC}$ and $\mathrm{OmpF}$ in Escherichia coli. Biosci Biotechnol Biochem 63, 408-414.

Miller, J. (1972). Experiments in Molecular Genetics. Cold Spring Harbor, NY : Cold Spring Harbor Laboratory.

Notley, L. \& Ferenci, T. (1996). Induction of RpoS-dependent functions in glucose-limited continuous culture: what level of nutrient limitation induces the stationary phase of Escherichia coli? J Bacteriol 178, 1465-1468.

Notley-McRobb, L., Death, A. \& Ferenci, T. (1997). The relationship between external glucose concentration and cAMP levels inside Escherichia coli: implications for models of phosphotransferase-mediated regulation of adenylate cyclase. Microbiology 143, 1909-1918.

Nystrom, T. (1998). To be or not to be - the ultimate decision of the growth-arrested bacterial cell. FEMS Microbiol Rev 21, 283-290.

Overbeeke, N. \& Lugtenberg, B. (1980). Expression of outer membrane protein e of Escherichia coli K12 by phosphate limitation. FEBS Lett 112, 229-232.

Painbeni, E., Caroff, M. \& Rouviere-Yaniv, J. (1997). Alterations of the outer membrane composition in Escherichia coli lacking the histone-like protein HU. Proc Natl Acad Sci US A 94, 6712-6717.

Pratt, L. A., Hsing, W., Gibson, K. E. \& Silhavy, T. J. (1996). From acids to osm $Z$ : multiple factors influence the synthesis of the $\mathrm{OmpF}$ and OmpC porins in Escherichia coli. Mol Microbiol 20, 911-917.

Ramani, N., Huang, L. \& Freundlich, M. (1992). In vitro interactions of integration host factor with the ompF promoter-regulatory region of Escherichia coli. Mol Gen Genet 231, 248-255.

Russo, F. D. \& Silhavy, T. J. (1991). EnvZ controls the concentration of phosphorylated OmpR to mediate osmoregulation of the porin genes. J Mol Biol 222, 567-580.

Scott, N. W. \& Harwood, C. R. (1980). Studies on the influence of the cyclic AMP system on major outer membrane proteins of Escherichia coli K12. FEMS Microbiol Lett 9, 95-98.

Shiga, Y., Kametani, S., Kadokura, T. \& Akanuma, H. (1999). 1,5Anhydroglucitol promotes glycogenolysis in Escherichia coli. J Biochem 125, 166-172.
Shin, S. \& Park, C. (1995). Modulation of flagellar expression in Escherichia coli by acetyl phosphate and the osmoregulator OmpR. J Bacteriol 177, 4696-4702.

Slauch, J. M. \& Silhavy, T. J. (1989). Genetic analysis of the switch that controls porin gene expression in Escherichia coli K-12. J Mol Biol 210, 281-292.

Slauch, J. M., Garrett, S., Jackson, D. E. \& Silhavy, T. J. (1988). EnvZ functions through OmpR to control porin gene expression in Escherichia coli K-12. J Bacteriol 170, 439-441.

Sterkenburg, A., Vlegels, E. \& Wouters, J. T. M. (1984). Influence of nutrient limitation and growth rate on the outer membrane proteins of Klebsiella aerogenes NCTC 418. J Gen Microbiol 130, 2347-2355.

Suzuki, T., Ueguchi, C. \& Mizuno, T. (1996). H-NS regulates ompF expression through micF antisense RNA in Escherichia coli. J Bacteriol 178, 3650-3653.

Teich, A., Meyer, S., Lin, H. Y., Andersson, L., Enfors, S. \& Neubauer, P. (1999). Growth rate related concentration changes of the starvation response regulators sigma(S) and ppGpp in glucose-limited fed-batch and continuous cultures of Escherichia coli. Biotechnol Prog 15, 123-129.

Thomas, A. D. \& Booth, I. R. (1992). The regulation of expression of the porin gene ompC by acid pH. J Gen Microbiol 138, 1829-1835.

Tokishita, S. \& Mizuno, T. (1994). Transmembrane signal transduction by the Escherichia coli osmotic sensor, EnvZ: intermolecular complementation of transmembrane signalling. Mol Microbiol 13, 435-444.

Tsui, P., Helu, V. \& Freundlich, M. (1988). Altered osmoregulation of ompF in integration host factor mutants of Escherichia coli. J Bacteriol 170, 4950-4953.

Tweeddale, H., Notley-McRobb, L. \& Ferenci, T. (1998). Effect of slow growth on metabolism of Escherichia coli, as revealed by global metabolite pool ('metabolome') analysis. J Bacteriol 180, 5109-5116.

Yamada, H., Yoshida, T., Tanaka, K., Sasakawa, C. \& Mizuno, T. (1991). Molecular analysis of the Escherichia coli hns gene encoding a DNA-binding protein, which preferentially recognizes curved DNA sequences. Mol Gen Genet 230, 332-336.

Yu, D. G., Ellis, H. M., Lee, E. C., Jenkins, N. A., Copeland, N. G. \& Court, D. L. (2000). An efficient recombination system for chromosome engineering in Escherichia coli. Proc Natl Acad Sci US A 97, 5978-5983.

Received 8 March 2001; revised 22 June 2001; accepted 23 July 2001. 